\title{
Quasi-Optical Design and Analysis of the Millimeter-Wave Bolometric Interferometer (MBI)
}

\author{
Gareth S. Curran, Marcin L. Gradziel, Creidhe O'Sullivan, J. Anthony Murphy, Peter O. Hyland, \\ Andrei Korotkov, Siddharth Malu, Lucio Piccirillo, Peter T. Timbie and Gregory S. Tucker
}

\begin{abstract}
This paper presents work being done on the optical design and analysis of the beam combiner for the MillimeterWave Bolometric Interferometer (MBI), a ground based instrument designed to measure the polarization anisotropies of the Cosmic Microwave Background at a central frequency of 90GHz. Interferometry has never before been used to carry out such measurements at this frequency, nor using incoherent bolometers as detectors. MBI will therefore act as a prototype for this type of system and must be modeled in detail to ensure that its operation is fully understood.
\end{abstract}

Index Terms-Analysis, cosmic microwave background, interferometer, quasi-optics, telescope, polarization.

\section{INTRODUCTION}

The Cosmic Microwave Background (CMB) is the relic radiation from the Big Bang and exhibits very faint temperature anisotropies indicative of the primordial density fluctuations of the early universe. The temperature power spectrum has been well characterized over a broad range of angular scales, however it is now realized that temperature measurements alone are not sufficient to uniquely constrain the cosmological constants required for detailed modeling. A more

Manuscript received June 18, 2007. This work was supported in part by Science Foundation Ireland under its Research Frontiers Program.

G. S. Curran is with the Institute of Technology Blanchardstown, Dublin 15, Ireland. (Tel: +353-1-8851097; fax: +353-1-8851001; e-mail: gareth.curran@itb.ie).

M. L. Gradziel is with the National University of Ireland Maynooth, Co. Kildare, Ireland. (e-mail: marcin.gradziel@nuim.ie).

C. O'Sullivan is with the National University of Ireland Maynooth, Co. Kildare, Ireland. (e-mail: creidhe.osullivan@nuim.ie).

J. A. Murphy is with the National University of Ireland Maynooth, Co. Kildare, Ireland. (e-mail: anthony.murphy@nuim.ie).

P. O. Hyland is with the University of Wisconsin-Madison, Madison, WI53706. (e-mail: pohyland@wisc.edu).

A. Korotkov is with Brown University, Providence, RI02912. (e-mail: andrei korotkov@brown.edu).

S. Malu is with the University of Wisconsin-Madison, Madison, WI53706. (e-mail: ssmalu@wisc.edu).

L. Piccirillo is with the University of Manchester, Manchester, M139PL, UK. (e-mail: lucio@jb.man.ac.uk).

P. Timbie is with the University of Wisconsin-Madison, Madison, WI53706. (e-mail: pttimbie@wisc.edu).

G. Tucker is with Brown University, Providence, RI02912. (e-mail: gregory_tucker@brown.edu). comprehensive analysis shows that there are anisotropies in the linear polarization of the $\mathrm{CMB}$ which are introduced by Thompson scattering of photons at re-ionization. This was first detected by the Degree Angular Scale Interferometer (DASI) experiment [1] in 2002. Accurate measurements of this polarization are expected to lead to tighter constraints on cosmological parameters describing the density, composition and expansion rate of the universe and the latest generation of experiments, such as QUaD [2], PLANK [3] and MBI [4], are only now approaching the sensitivity needed to carry out these measurements.

MBI is a ground-based telescope designed to make measurements of the polarization of the $\mathrm{CMB}$ at degree angular scales at a frequency of $90 \mathrm{GHz}$. It is a Fizeau interferometer that uses cooled spider web bolometers for detectors, combining the advantages of interferometry for control of systematic effects with the high sensitivity and frequency coverage possessed by bolometers [5]. It is the first bolometric interferometer of its kind which results in a novel instrument with capabilities that would be difficult to achieve using more traditional techniques and will allow MBI and instruments based on its technology to explore a wide range of angular scales and wavelengths. This novel approach demands detailed modeling and analysis of the telescope not only to prove the concept of operation but also to account for any systematic effects that may arise during observations. In this paper we deal specifically with the front-end optics of the beam combiner as described in the next section.

\section{THE TELESCOPE}

MBI-4, the first generation of the instrument, is an adding interferometer that views the CMB through 4 corrugated conical horn antennas. Each antenna selects a single linear polarization and the signals are correlated using a cryogenic beam-combining telescope (Fizeau combiner) consisting of a parabolic primary mirror and a hyperbolic secondary mirror. The signals from each baseline form an interference fringe pattern in the focal plane on an array of 19 spider-web bolometers fed by conical horns. Phase-modulation of the signal from each antenna modulates the fringes in the focal plane and allows lock-in detection of the visibilities [6]. 


\section{ANALYSIS TECHNIQUES AND RESULTS}

The first step in the analysis of the MBI system involved calculating the aperture field produced by the corrugated horn antennas that illuminate the primary mirror. This was achieved using NUI Maynooth's modeling software, SCATTER [7], which assumes that the horn structure is composed of a series of cylindrical waveguide sections and uses a rigorous electromagnetic mode-matching technique to calculate the power scattered between propagating modes at each waveguide junction. In many applications a conical corrugated horn can be well approximated by a truncated Bessel function. This is the case for the corrugated conical horns used in MBI4 and using this approximation increases the computational efficiency during analysis.

To calculate the power coupling between each of the 4 feed horns and the 19 detector horn antennas, a physical optics analysis was carried out using MODAL [8]. Results from this calculation showed that the coupled power was quite low compared to the original power injected into the system and therefore it was necessary to locate those positions in the system where the power was being truncated. Table I shows the fraction of the source power $(1 \mathrm{~W})$ at different planes throughout the system. It can be clearly seen that most of the power is being lost at transmission through the elliptical aperture of the primary mirror after reflection at the secondary mirror. To demonstrate this further the electric field was calculated at the detector plane both with and without this truncation effect. As shown in Fig. 2, the interference pattern obtained when truncation at the primary mirror is neglected is far more ideal than that when truncation is taken into account.

\section{DISCUSSION AND CONCLUSIONS}

A full analysis of the MBI-4 system, including the back to back feed horn structures and the bolometers, remains to be carried out. Following this we will look to improve the system for a future version of MBI as a space spaced instrument. However, the concept of operation of $\mathrm{MBI}$ as an interferometer has been demonstrated.

\section{REFERENCES}

[1] J. Kovac, E.M. Leitch, C. Pryke, J.E. Carlstrom, N.W. Halverson, W.L. Holzapfel, "Detection of polarization in the cosmic microwave background using DASI", Nature, 420, 772, 2002.

[2] Church S. et al., "QUEST on DASI: a South-Pole CMB polarization experiment", New Astron. Rev., 47, 1083-1089, 2003.

[3] J.-M. Lamarre et al., "The Planck High-Frequency Instrument, a Third generation CMB Experiment, and a Full-Sky Submillimeter Survey", New Astron. Rev., 47, 1017-1024, 2003.

[4] P. T. Timbie, G. S. Tucker, P. A. R. Ade, S. Ali, E. Bierman, E. F. Bunn, C. Calderon, A. C. Gault, P. O. Hyland, B. G. Keating, J. Kim, A. Korotkov, S. S. Malu, P. Mauskopf, J. A. Murphy, C. O’Sullivan, L. Piccirillo, B. D. Wandelt, "The Einstein polarization interferometer for cosmology (EPIC) and the millimeter-wave bolometric interferometer (MBI)", New Astron. Rev., 50, 999-1008, 2006.

[5] A. Korotkov, J. Kim, G. S. Tucker, A.C. Gault, P.O. Hyland, S. S. Malu, P. T. Timbie, E. Bunn, E. Bierman, B. Keating, J. A. Murphy, C. O'Sullivan P. A. R. Ade, C. Calderon, L. Piccirillo, "The MillimeterWave Bolometric Interferometer", SPIE 6273.

[6] C. O’Sullivan, J. A. Murphy, V. Yurchenko, G. Cahill, G. S. Curran, M. L. Gradziel, J. Lavelle, F. Noviello, "The Quasi-Optical Performance of CMB Astronomical Telescopes” Proc. SPIE Vol.6472, 647202.
[7] J. A. Murphy, R. Colgan, C. O’Sullivan, B. Maffei, P.A.R. Ade, "Radiation patterns of multi-moded corrugated horns for far-IR space applications", Infrared Physics and Technology, 42, pp 515-528, 2001.

[8] M. L. Gradziel, C. O’Sullivan, J.A. Murphy, G. Cahill, G.S. Curran, C. Pryke, W. Gear, S. Church, "Modeling of the optical performance of millimeter-wave instruments using MODAL", Proc. SPIE Vol.6472, 64720D.

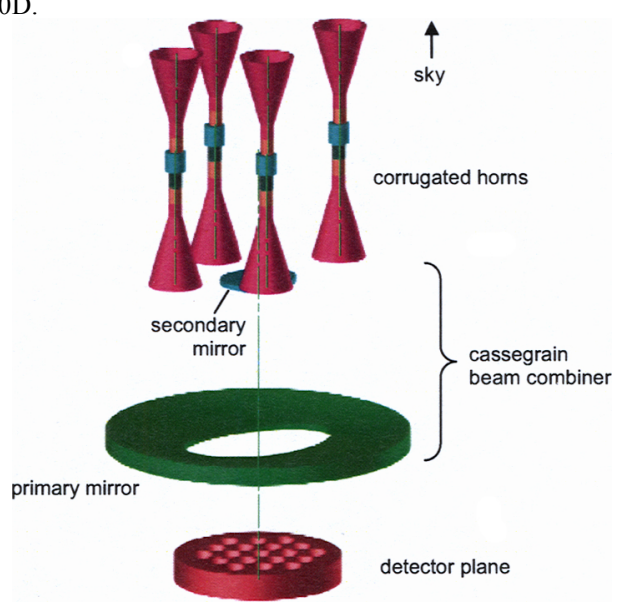

Fig. 1. Schematic diagram of the MBI-4 telescope.

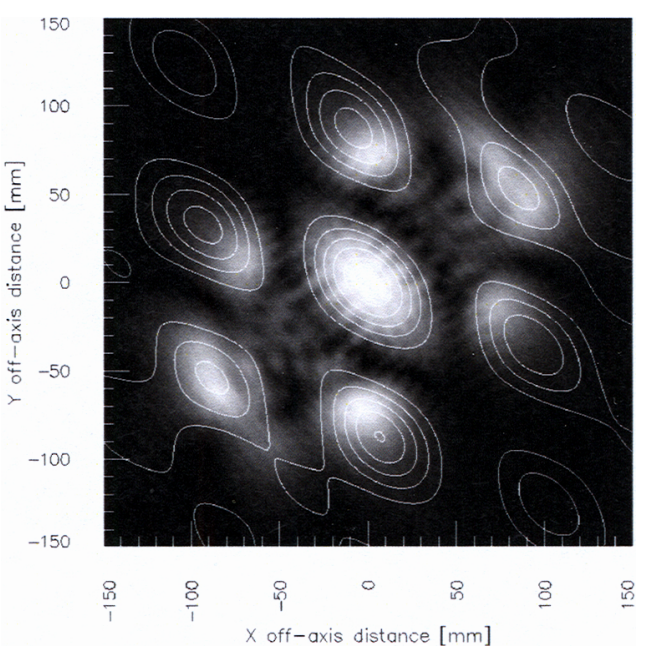

Fig. 2. The field at the image plane of the telescope. The contours show the intensity with no truncation at the primary taken into account while the colour map takes into account this truncation. These results assume that the sources are in phase and are linearly polarised in the same direction.

Table I

Power in the Telescope

\begin{tabular}{|l|c|c|c|c|}
\hline \multirow{2}{*}{ Point in System } & \multicolumn{4}{|c|}{ Power (W) } \\
\cline { 2 - 5 } & Source 1 & Source 2 & Source 3 & Source 4 \\
\hline Source & 1 & 1 & 1 & 1 \\
\hline Sec_Trans & 0.999542 & 0.999551 & 0.999624 & 0.999624 \\
\hline Primary Reflect & 0.894243 & 0.835838 & 0.920831 & 0.921858 \\
\hline Sec_Reflect & 0.86939 & 0.804995 & 0.889668 & 0.890841 \\
\hline Prim_Trans & 0.273388 & 0.233216 & 0.29192 & 0.292775 \\
\hline Image & 0.269604 & 0.229971 & 0.28785 & 0.2887 \\
\hline
\end{tabular}

The power (W) at a series of elements in the system: source, secondary transmission (on the way towards primary), primary after reflection, secondary after reflection, primary after truncation and image plane. 